\title{
Using Three-Dimensional Electron Backscatter Diffraction Data to Measure Grain Boundary Properties in Metals and Ceramics
}

\author{
Gregory S. Rohrer ${ }^{1}$ \\ ${ }^{1}$.Department of Material Science and Engineering, Carnegie Mellon University, Pittsburgh, PA, USA
}

A Dual Beam (DB) Focused Ion Beam (FIB) Scanning Electron Microscope (SEM) is an instrument that combines all of the functionality of an SEM for imaging structure, composition, and orientation, with the ability to remove material in a highly controlled fashion and with nano-scale precision. It can be stated without exaggeration that the DB-FIB SEM has revolutionized materials characterization by enabling experiments that were not before possible. For example, by analyzing a surface with the SEM, and then systematically removing parallel layers with the ion beam, it is now possible to visualize the internal structure and composition of three-dimensional volumes of opaque material with a resolution in the nanometer range.

The grain boundary character distribution represents the relative areas of grain boundaries in a polycrystal as a function of lattice misorientation and grain boundary plane orientation. Analogous distributions can be determined for the grain boundary energy and curvature as a function of grain boundary crystallography. Measuring these distributions requires precision serial sectioning coupled with electron backscatter diffraction (EBSD) mapping; because of the difficulty of this procedure, there were only a few measurements reported before 2009. However, the introduction of the DB-FIB SEM with a liquid metal Ga-ion source accelerated the pace of such measurements, because the serial sectioning could be conducted automatically, in situ $[1,2]$. The pace of these measurements has increased with the recent introduction of the Xe-plasma FIB [3]. These 3D measurements have led to a new understanding of what controls grain boundary (GB) distributions in polycrystals. For example, it has been found that GB properties vary significantly as a function of GB plane orientation, more so than with misorientation. Variations in GB energy as a function of GB plane orientation are far more significant in determining GB populations than lattice coincidence. Also, during microstructural evolution, high-energy GBs are preferentially eliminated from the distribution. This leads to the development of a predictable distribution of grain boundary types [4].

This paper will describe recent measurements of grain boundary populations, energies, and curvatures as a function of all five crystallographic parameters. As an example, the curvature as a function of the grain boundary plane orientation for grain boundaries with a $60^{\circ}$ disorientation about the [111] axis $(\Sigma 3)$ for an austenite and ferrite sample are illustrated in Fig. 1 [5]. The relative area, energy, and curvature are plotted in stereographic projection with [001] and [100] pointing normal to the plane of the paper and to the right, respectively $[1,2,5]$. For the austenite sample, the most significant feature appears at the position of the coherent twin. This is the minimum curvature, maximum population, and minimum energy. This is the boundary that is terminated by (111) planes on both sides and is very flat. It is interesting to note that there is a maximum in curvature for boundaries that are $90^{\circ}$ from the twin position and the maximum is reached at the positions of the (110) boundary planes. This is consistent with the observation that twined grains are usually plate-shaped and have large parallel $\{111\}$ faces connected by much smaller perpendicular boundaries. The high curvature results from geometric necessity of joining the two parallel $\{111\}$ planes to bound the grain. The distributions for the ferritic sample are nearly opposite that of the austenitic sample. The lowest curvatures occur in the [111] zone 
with the minimum curvature, energy, and maximum population occurring at the positions of the $\{211\}$ symmetric tilt grain boundaries.

The results show that grain boundary energies and curvatures can be accurately measured from discrete FIB data. We find that the crystallography of the grain boundary strongly influences the curvature. The variation of the curvature with the grain boundary plane orientation is stronger than the variation with grain boundary disorientation angle. The lowest curvature grain boundaries also have the lowest grain boundary energies and highest grain boundary areas.

\section{References:}

[1] H. Beladi and G.S. Rohrer, Acta Mater., 61 (2013) 1404.

[2] H. Beladi, N.T. Nuhfer, and G.S. Rohrer, Acta Mater., 70 (2014) p. 281.

[3] M.N. Kelly, K. Glowinski, N.T. Nuhfer, and G.S. Rohrer, Acta Mater., 111 (2016) 22.

[4] G.S. Rohrer, J. Mater. Sci., 46 (2011) 5881.

[5] X. Zhong, D.J. Rowenhorst, H. Beladi, and G.S. Rohrer, Acta Mater., 123 (2017) 136
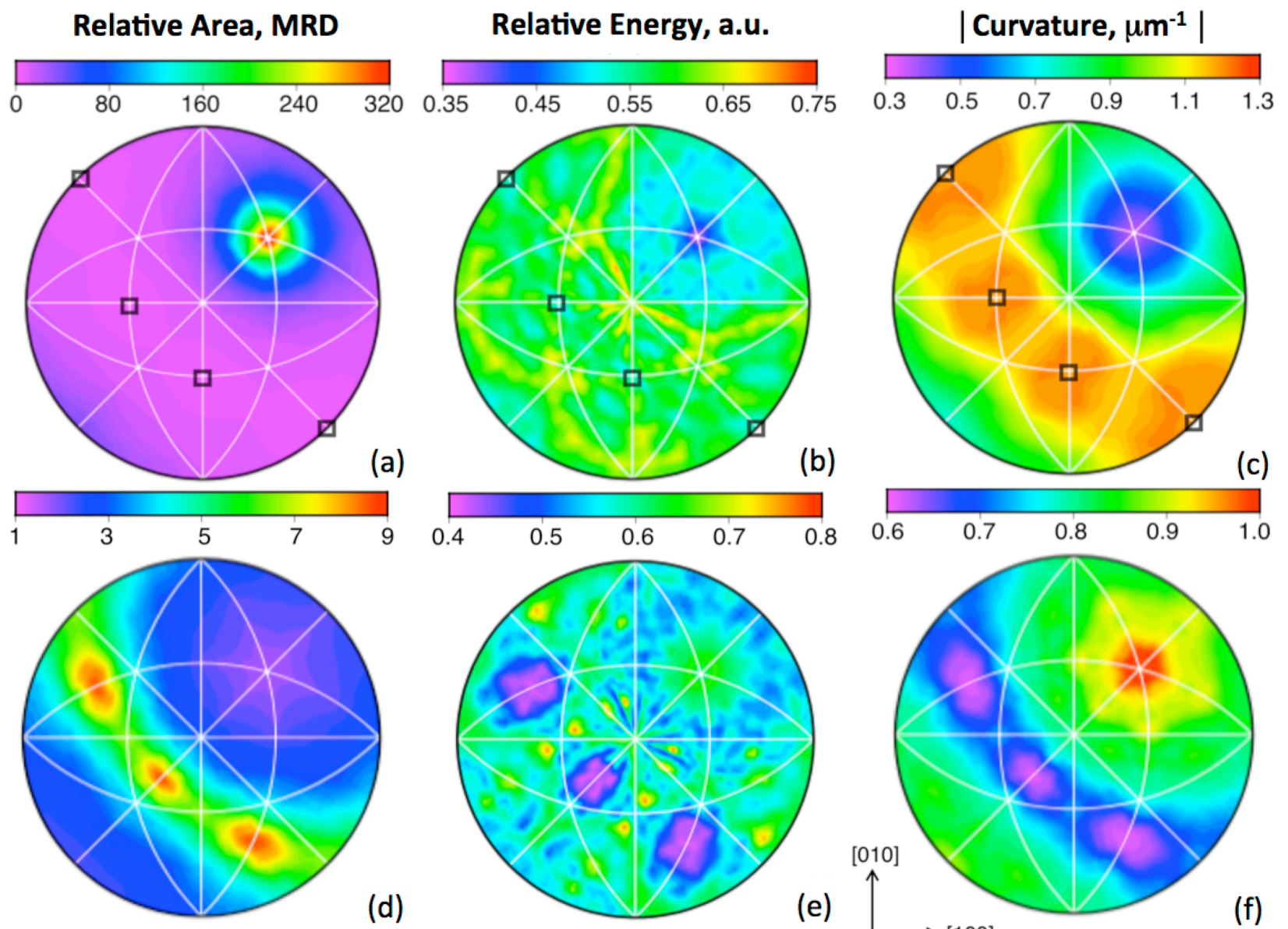

(b)
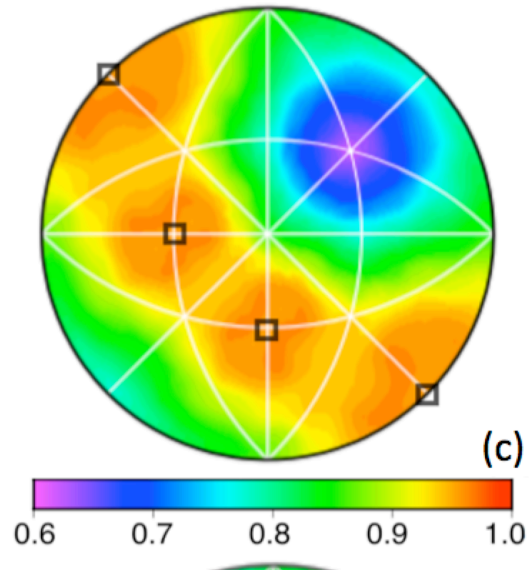

(e)

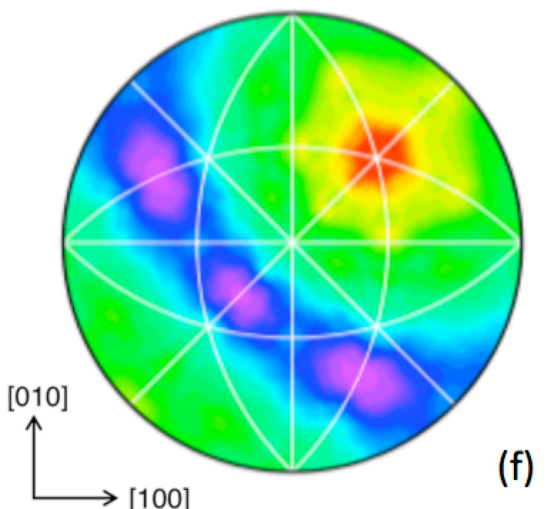

Figure 1. Population (a,d), energy (b, e), and curvature (c, f) distributions for austenite (a-c) and ferrite (d-f) for all grain boundaries with a $\Sigma 3(60 \%[111])$ disorientation. The small squares are for reference, marking $\{110\}$ locations in the [111] zone in (a-c) and are omitted from (d-f) for clarity. 